\title{
Dependence of Structural and Electronic Properties of Uranium Monochalcogenides on Exchange-Correlation Energy Functionals
}

\author{
Shugo Suzuki, Hidehisa Ohta, Takumi Komatsu, and Sho Yasuda \\ Institute of Materials Science, University of Tsukuba, Tsukuba 305-8573
}

(Received June 13, 2011)

\begin{abstract}
We study the dependence of the structural properties of uranium monochalcogenides, $\mathrm{U} X$ where $X=\mathrm{S}$, Se, and Te, as well as their electronic ones on the exchange-correlation energy functionals within the spin density functional theory, carrying out all electron calculations by the fully relativistic full-potential linear-combination-of-atomic-orbitals method. We employ two functionals of the local spin density approximation (LSDA) and two functionals of the generalized gradient approximations (GGA); the former two are the Perdew-Zunger and Perdew-Wang functionals and the latter two are the Perdew-Burke-Ernzerhof (PBE) and PBEsol functionals. We also examine the effects of the relativistic correction to the LSDA exchange part of each functional. We find that, for lattice constants, bulk moduli, and cohesive energies, the results of the calculations using the PBE functional are in the best agreement with the experimental results. On the contrary, we find that calculated total magnetic moments and one-electron energies are almost the same for all the LSDA and GGA functionals employed in this work, failing to improve the agreement between the calculated and experimental results even if the gradient and relativistic corrections are included. We also find that the relativistic correction plays minor roles in both the structural and electronic properties.
\end{abstract}

KEYWORDS: uranium monochalcogenide, spin density functional theory, exchange-correlation energy functional, local spin density approximation, generalized gradient approximation

\section{Introduction}

Over the past few decades, the structural and electronic properties of the materials with actinide elements have been studied extensively from both the experimental and theoretical sides. ${ }^{1)}$ In particular, the recent progress in the computational techniques in first-principles calculations has enable us to study their structural properties in detail: ${ }^{2)}$ fully relativistic calculations for taking account of not only scalar relativistic effects but also spin-orbit coupling, full-potential calculations for evaluating the electrostatic energy and potential accurately without resorting a shape approximation, and the generalized gradient approximation (GGA) for improving calculated exchange-correlation energies. Even when restricted to actinide metals and AnX-type compounds, a number of theoretical studies have been made on their structural properties, applying these advanced methods. ${ }^{3-12)}$ 
A series of early studies by Brooks and his co-workers made important contributions to qualitative understanding of the trends in the structural properties of the materials with actinide elements. ${ }^{13-19)}$ In their studies, the lattice constants and the bulk moduli were calculated using an approximate equation of state with the assumption of paramagnetic ground states. In spite of several approximations employed, the overall properties have been explained successfully. Subsequently, several theoretical studies have been carried out using the fully relativistic and full-potential calculations with GGA. ${ }^{3-12)}$ Soderlind et al. ${ }^{3)}$ have studied the structural properties of actinide metals; they have shown that the PW91 functional, which is a GGA functional introduced by Perdew et al. ${ }^{20,21)}$ works well when replacing the local spin density approximation (LSDA) with this functional. Furthermore, in the last decade, the structural properties of actinide nitrides and oxides have been studied extensively employing the PW91 functional and its simplified version, the PBE functional, which was proposed by Perdew, Burke, and Ernzerhof. ${ }^{22)}$ Consequently, it was shown that both the PW91 and PBE functionals also works well for calculating the structural properties of actinide nitrides and oxides.

Recently, the PBEsol functional ${ }^{23}$ ) has been proposed as a modified version of the PBE functional; the PBEsol functional is designed so as to work better for solids. However, a subsequent work by Söderlind et al. ${ }^{12)}$ has shown that the PBEsol functional drastically worsens the equilibrium properties of actinide metals compared to the PBE functional and produces results closer to those of LSDA. This is in accordance with the conclusion of another recent study ${ }^{24)}$ in which several types of exchange-correlation energy functionals were examined on a large set of solids with light elements and it was shown that the best exchange-correlation energy functional depends on the material; for example, LSDA is the best for gray tin while the PBE functional is the best for strontium metal in reproducing their lattice constants. Unfortunately, the PBEsol functional is not always better than other functionals.

Thus, it may be useful to study systematically the dependence of the structural properties of actinide compounds on the exchange-correlation energy functionals as was already done for actinide metals. ${ }^{12)}$ For this purpose, the actinide compounds to be studied first may be uranium monochalcogenides, $\mathrm{U} X$ where $X=\mathrm{S}$, Se, and Te, because they have a simple crystal structure and a simple electronic ground state, i.e., the $\mathrm{NaCl}$ structure and the ferromagnetic ground state. Although the electronic properties of UX have been studied extensively, ${ }^{25-33)}$ to our knowledge, there is only one first-principles study of the structural properties of $U X{ }^{25)}$ However, since GGA was not examined in that study, the dependence of the structural properties of $U X$ on the exchange-correlation energy functionals is still not clear.

The purpose of this work is to study the dependence of the structural properties of $\mathrm{U} X$ as well as their electronic ones on the exchange-correlation energy functionals within the spin density functional theory, carrying out all electron calculations by the fully relativistic 
full-potential linear-combination-of-atomic-orbitals (FFLCAO) method $^{34)}$ as well as by the scalar relativistic full-potential linear-combination-of-atomic-orbitals (SFLCAO) method ${ }^{35)}$ for comparison. We employ two LSDA and two GGA functionals; the former two are the Perdew-Zunger ${ }^{36)}$ and Perdew-Wang ${ }^{37)}$ functionals, both of which parameterize the CeperlyAlder results, ${ }^{38)}$ and the latter two are the $\mathrm{PBE}^{22)}$ and $\mathrm{PBEsol}^{23)}$ functionals. We also examine the effects of the relativistic correction to the LSDA exchange part of each functional. ${ }^{39)} \mathrm{We}$ describe the method of calculations in $\S 2$. The results and discussion are given in $\S 3$. Finally, we give the conclusions of this work in $\S 4$.

\section{Method of Calculations}

We carried out all electron calculations using the FFLCAO method ${ }^{34)}$ as well as the SFLCAO method ${ }^{35)}$ for comparison. In the FFLCAO method, a single-particle equation of the Kohn-Sham-Dirac type is solved not only for core orbitals but also for valence orbitals. In the SFLCAO method, on the other hand, both core and valence orbitals are calculated through a procedure for averaging spin-orbit coupling. ${ }^{35)}$ As already mentioned, we employed two LSDA and two GGA functionals; the former two are the Perdew-Zunger ${ }^{36)}$ and Perdew-Wang ${ }^{37)}$ functionals, both of which parametrize the Ceperly-Alder results, ${ }^{38)}$ and the latter two are the $\mathrm{PBE}^{22)}$ and $\mathrm{PBEsol}{ }^{23)}$ functionals. We also study the effects of the relativistic correction to the LSDA exchange part of each functional. ${ }^{39)}$ We carried out self-consistent calculations using the exchange-correlation potentials derived from the corresponding exchange-correlation energy functionals; the atomic orbitals were generated in advance using the same exchangecorrelation potential as that used in the self-consistent calculations of $\mathrm{U} X$.

It is worth mentioning that employing both the Perdew-Zunger and Perdew-Wang functionals, instead of one of them, may be justified because of the following reasons although these two functionals are the parameterizations of the same numerical results. Firstly, since the Perdew-Zunger functional is one of the most widely used LSDA functionals, it is useful to show the results of the calculations employing this parameterization. Secondly, since the Perdew-Wang functional is designed so as to fit the Ceperly-Alder results better than the Perdew-Zunger functional, it is reasonable to use the Perdew-Wang functional, examining how large the difference between the two functionals is. Thirdly, since the Perdew-Wang functional is the one that is routinely employed as the LSDA part of the PBE and PBEsol functionals, it is important to study the effects purely originated in the inclusion of the gradient corrections comparing the results obtained using the Perdew-Wang functional and those obtained using the PBE or PBEsol functional.

$\mathrm{UX}$ crystallizes in the $\mathrm{NaCl}$ structure exhibiting a strong magnetic anisotropy with an easy axis in the [111] direction. ${ }^{1)}$ The experimental lattice constants of US, USe, and UTe are 5.489, 5.740, and $6.155 \AA$, respectively. ${ }^{40)}$ We assumed that the magnetization axis is in the [111] direction, which was taken as the $z$ axis in our calculations. The basis functions adopted 
in the FFLCAO and SFLCAO methods consist of the following atomic orbitals: $1 s, 2 s, 2 p$, $3 s, 3 p, 3 d, 4 s, 4 p, 4 d, 4 f, 5 s, 5 p, 5 d, 5 f, 6 s, 6 p, 6 d$, and $7 s$ orbitals of the neutral $\mathrm{U}$ atom, $5 f, 7 s$, and $7 p$ orbitals of the $\mathrm{U}^{2+}$ atom, $1 s, 2 s, 2 p, 3 s$, and $3 p$ atomic orbitals of the neutral $\mathrm{S}$ atom, and $3 s, 3 p$, and $3 d$ orbitals of the $\mathrm{S}^{2+}$ atom, $1 s, 2 s, 2 p, 3 s, 3 p, 3 d, 4 s$, and $4 p$ atomic orbitals of the neutral Se atom, and $4 s, 4 p$, and $4 d$ orbitals of the $\mathrm{Se}^{2+}$ atom, $1 s, 2 s, 2 p$, $3 s, 3 p, 3 d, 4 s, 4 p, 4 d, 5 s$, and $5 p$ atomic orbitals of the neutral Te atom, and $5 s, 5 p$, and $5 d$ orbitals of the $\mathrm{Te}^{2+}$ atom. It is important to note that the use of not only the atomic orbitals of neutral atoms but also those of positively charged atoms is crucial to the description of the contraction of atomic orbitals associated with cohesion. We carried out real-space integration using 4644 points for the U atom, 2580 points for the S atom, 3096 points for the Se atom, and 3612 points for the Te atom. The Brillouin-zone integration was carried out using the good-lattice-point method with $185 \boldsymbol{k}$ points. ${ }^{41)}$

The total energy of $U X$ was calculated as a function of lattice constant. Then, the lattice constant, the bulk modulus, and the cohesive energy in the optimized structure were evaluated using Murnaghan's equation of state; ${ }^{42)}$ the cohesive energy was obtained subtracting the sum of the total energies of the isolated $\mathrm{U}$ and $X$ atoms from the total energy of $\mathrm{U} X$, where the same exchange-correlation energy functional and potential were employed. We also study the orbital, spin, and total magnetic moments per unit formula, $M_{\mathrm{orb}}, M_{\mathrm{spin}}$, and $M_{\mathrm{tot}}$, using the experimental lattice constants. The reason for the use of the experimental lattice constants is that we are interested in their dependence purely on the exchange-correlation energy functionals, not on the optimized lattice constants which differ for different exchange-correlation energy functionals. The magnetic moments were calculated using the method described in our previous work. ${ }^{32}$ ) In this method, space is partitioned into atomic Voronoi cells and the total magnetic moment of each atomic Voronoi cell is calculated integrating the cross product between the position vector and the Dirac current over the atomic Voronoi cell. Then, the orbital magnetic moment is calculated subtracting the spin magnetic moment from the total magnetic moment, where the spin magnetic moment is obtained integrating the spin density over the atomic Voronoi cell.

\section{Results and discussion}

We begin with the results of the optimization of the lattice constants. The results of the FFLCAO and SFLCAO calculations are listed in Table I; those in parentheses are the results of the SFLCAO calculations. In the table, the experimental lattice constants ${ }^{40)}$ are also shown. For each material, the first and second rows show the results of the calculations without and with the relativistic correction to the LSDA exchange part, respectively. It is found that the agreement of the results obtained using the PBE functional with the experimental lattice constants is the best; the errors are about $+0.5 \%$. The next best is the PBEsol functional; the errors are about $-1 \%$. Although the Perdew-Zunger and Perdew-Wang results are in 
agreement with each other, the agreement with the experimental results are the worst; the errors are about $-2 \%$. It is worth mentioning that the relativistic correction to the LSDA exchange part affects the results very little; this indicates that the orbitals mainly affected by the relativistic correction are not the valence orbitals but the core orbitals. We also note that the results of the FFLCAO and SFLCAO calculations are in good agreement with each other, indicating that the procedure for averaging spin-orbit coupling in the SFLCAO method works well. $^{35)}$

We next examine the calculated bulk moduli. The results of the FFLCAO and SFLCAO calculations are listed in Table II; those in parentheses are the results of the SFLCAO calculations. In the table, the experimental bulk moduli43) are also shown. For each material, the first and second rows show the results of the calculations without and with the relativistic correction to the LSDA exchange part, respectively. We find that the PBE functional is the best; the errors are about 10, 30, and $45 \%$ for US, USe, and UTe, respectively. The next best is the PBEsol functional; the errors are about 25, 45, and $70 \%$ for US, USe, and UTe, respectively. The Perdew-Zunger and Perdew-Wang functionals are the worst; the errors are about 35,60 , and $80 \%$ for US, USe, and UTe, respectively. It is also found that there is a tendency of slight reduction in the bulk moduli when the relativistic correction to the LSDA exchange part is included. We again find that the results of the FFLCAO and SFLCAO calculations agree reasonably with each other.

The results of the FFLCAO and SFLCAO calculations of the cohesive energies are listed in Table III; those in parentheses are the results of the SFLCAO calculations. In the table, the experimental cohesive energies ${ }^{44)}$ are also shown. For each material, the first and second rows show the results of the calculations without and with the relativistic correction to the LSDA exchange part, respectively. The PBE functional is the best; the errors are about $15 \%$. The next best is the PBEsol functional; the errors are about $25 \%$. The Perdew-Zunger and Perdew-Wang results are the worst; the errors are about $30 \%$. The inclusion of the relativistic correction to the LSDA exchange part affects the results very little, indicating again that the relativistic correction is not very important to the valence orbitals. We also note that the agreement between the FFLCAO and SFLCAO results is good.

We thus find that the PBE functional is the best among the functionals employed in this work in calculating the lattice constants, the bulk moduli, and the cohesive energies. In particular, the lattice constants calculated using the PBE functional are in excellent agreement with the experimental ones with the errors of less than $1 \%$. It should be noted, however, that the PBE functional still overestimates the bulk moduli and the cohesive energies although the results are improved considerably in comparison with those calculated using the other three functionals; for example, the bulk moduli of UTe calculated using this functional is larger than the experimental one by about $45 \%$. 
So far, to our knowledge, there is only one first-principles study of the lattice constants and the bulk moduli ${ }^{25)}$ the calculations were carried out for US and UTe using a full-potential linear muffin-tin-orbital method, where spin-orbit coupling is included at each variational step, with the von Barth-Hedin expression of the LSDA exchange-correlation energy functional. ${ }^{45}$ ) The optimized lattice constants of US and UTe are 5.30 and $5.95 \AA$, respectively. These values are slightly smaller than our results for US and UTe, 5.37 and $6.04 \AA$. Their estimated bulk moduli of US and UTe at the optimized lattice constants are 130 and $109 \mathrm{GPa}$, respectively, which are to be compared with our Perdew-Zunger (Perdew-Wang) results for US and UTe, 143 (142) and 88 (87) GPa. The former is larger than their result by about 10 GPa while the latter is smaller than their result by about $20 \mathrm{GPa}$ although the origin of the discrepancy is not clear. One possible origin is the difference in the employed LSDA functional. On the other hand, the approximation used in their calculations for including spin-orbit coupling, i.e., the second variational method, may not be the origin of the discrepancy if the scalar relativistic effects are taking into account appropriately, as indicated by the good agreement between our results of the FFLCAO and SFLCAO calculations.

It may be worth discussing the functional dependence of the structural properties of $\mathrm{U} X$ by comparing it with that of other materials. For example, it has been shown that the PBE functional is not very good for calculating the lattice constants of $\mathrm{Pt}, \mathrm{Au}$, and $\mathrm{Pb} ;{ }^{24}$ the Perdew-Wang functional is the best for Pt and $\mathrm{Au}$ and the PBEsol functional is the best for $\mathrm{Pb}$. This means that the PBE functional is not always the best for the materials with heavy elements. What are the characteristics that control the functional dependence of the actinide materials? One may consider that it is the existence of the $5 f$ electrons. However, this is not true because a functional dependence similar to that of $U$ has been found for Th, which is an actinide element with no $5 f$ electrons. ${ }^{12,24)}$ We may find a key to understand the functional dependence of the actinide materials by comparing it with that of the alkali/alkali-earth metals. For the alkali/alkali-earth metals, the PBE or PBEsol functional works much better than the Perdew-Wang functional. ${ }^{24)}$ There are two common features of the alkali/alkaliearth and actinide elements. One is that they have the valence electrons with large spatial extension, i.e., the $n s$ electrons where $n=2-6$ for the alkali/alkali-earth elements and the $6 d$ and $7 s$ electrons for the actinide elements. The other is that the semicore electrons, i.e., the $n-1 p$ electrons for the alkali/alkali-earth elements and the $6 p$ electrons for the actinide elements, have much lower one-electron energies than the semicore electrons in $\mathrm{Pt}, \mathrm{Au}$, and $\mathrm{Pb}$, i.e., the $5 d$ electrons. These two features may be closely related to the functional dependence of the structural properties of the materials with the alkali/alkali-earth metals and the actinide materials. Further detailed studies are indispensable in the future to elucidate the origin of the functional dependence of the structural properties of individual materials.

We now study $M_{\text {orb }}, M_{\text {spin }}$, and $M_{\text {tot }}$ calculated using the FFLCAO method, with the 
lattice constant of each material fixed at the experimental value. We did not carry out the SFLCAO calculations because $M_{\text {orb }}$ calculated using the SFLCAO method is completely zero due to the implicit treatment of spin-orbit coupling so that the comparison with the experimental results is meaningless. The results are listed in Table IV. In the table, the experimental $M_{\text {tot }}{ }^{40)}$ are also shown. For each material, the first and second rows show the results of the calculations without and with the relativistic correction to the LSDA exchange part, respectively. It is found that $M_{\text {tot }}$ is almost the same with each other regardless of the employed functionals; the difference is at most $0.03 \mu_{\mathrm{B}}$. Thus, the difference between the calculated and measured $M_{\text {tot }}$, about $0.5 \mu_{\mathrm{B}}$, cannot be explained even if the gradient and relativistic corrections are included. However, it should be noted that $M_{\text {orb }}$ and $M_{\text {spin }}$ increase in magnitude when LSDA is replaced with GGA; for example, both $M_{\text {orb }}$ and $M_{\text {spin }}$ for UTe calculated using the PBE functional are larger in magnitude by about $0.2 \mu_{\mathrm{B}}$ than those calculated using the Perdew-Zunger or Perdew-Wang functional. Nevertheless, $M_{\text {tot }}$ is almost unchanged because of the cancellation between the increases in $M_{\text {orb }}$ and $M_{\text {spin }}$.

There have been several attempts for improving the calculated magnetic moments; the inclusion of the orbital polarization (OP) correction ${ }^{18,25,28)}$ and the LDA $+U$ approach $^{30,33)}$ are two promising methods employed in the previous studies. It is shown that the OP correction enhances both $M_{\text {orb }}$ and $M_{\text {spin }}$ drastically, resulting in a better agreement of the calculated $M_{\text {tot }}$ with the measured one. However, the individual magnitudes of the calculated $M_{\text {orb }}$ and $M_{\text {spin }}$ are too large. ${ }^{30)}$ On the other hand, the $\mathrm{LDA}+U$ approach can give a good agreement of the calculated $M_{\mathrm{orb}}, M_{\text {spin }}$, and $M_{\text {tot }}$ with the measured ones if the on-site Coulomb repulsion $U$ is chosen appropriately. Unfortunately, there is an inconsistency in choosing an appropriate $U$ that can give a good agreement of both the calculated magnetic moments and the calculated lattice constants with the measured ones. ${ }^{33)}$ Thus, although the inclusion of the OP correction and the $\mathrm{LDA}+U$ approach are successful to a considerable degree, there remain some difficulties to be overcome in both the methods. Further theoretical studies are necessary in the future for more accurate calculations of the magnetic moments in $\mathrm{U} X$.

Finally, we examine the functional dependence of the one-electron energies. To this end, we calculated the X-ray absorption spectroscopy (XAS) spectra and the X-ray magnetic circular dichroism (XMCD) spectra at the $\mathrm{U} M_{4,5}$ edges, which are originated in the $\mathrm{U} 3 d \rightarrow 5 f$ transitions. When the relativistic correction is not included, we found that the resultant spectra are almost the same as those obtained in our previous work ${ }^{31)}$ even if the gradient correction is included; when we include the gradient correction, a slight enhancement is observed in the XMCD spectra while there are no noticeable changes in the XAS spectra. On the other hand, when the relativistic correction is included, we found that both the XAS and XMCD spectra shift to lower energy by about $8 \mathrm{eV}$; however, the overall shapes of the spectra are almost unchanged, indicating that the one-electron wave functions of the core and valence orbitals 
are affected by the inclusion of neither the gradient nor relativistic corrections. The origin of this energy shift is that, when the relativistic correction is included, the one-electron energies of the $\mathrm{U} 3 d$ orbitals shift to higher energy by about $8 \mathrm{eV}$ while the one-electron energies of the $\mathrm{U} 5 f$ orbitals are almost unchanged. These results indicate that the one-electron energies are almost unchanged even if the gradient and relativistic corrections are included except that the one-electron energies of the $\mathrm{U}$ core orbitals increase considerably by the inclusion of the relativistic correction.

\section{Conclusions}

We have studied the dependence of the structural and electronic properties of uranium monochalcogenides, $\mathrm{U} X$ where $X=\mathrm{S}$, Se, and Te, as well as their electronic ones on the exchange-correlation energy functionals. Using the FFLCAO method as well as the SFLCAO method for comparison, we carried out all electron calculations. The exchange-correlation energy functionals employed in this work are two LSDA and two GGA functionals; the former two are the Perdew-Zunger and Perdew-Wang functionals, both of which parametrize the Ceperly-Alder results, and the latter two are the PBE and PBEsol functionals. We also examined the effects of the relativistic correction to the LSDA exchange part of each functional. We found that, for lattice constants, bulk moduli, and cohesive energies, the results of the calculations using the PBE functional are in the best agreement with the experimental results. In particular, the lattice constants calculated using the PBE functional are in excellent agreement with the experimental ones with the errors of less than $1 \%$. However, the PBE functional still overestimates the bulk moduli and the cohesive energies although the results are improved considerably in comparison with those calculated using the other three functionals. We found that the relativistic correction does not affect the structural properties notably. We also found that the structural properties calculated using the FFLCAO and SFLCAO methods are in good agreement with each other, indicating that scalar relativistic calculations can reproduce the structural properties of the materials with heavy elements if spin-orbit coupling is taken into account through an appropriate averaging procedure. Furthermore, we have studied the dependence of the orbital, spin, and total magnetic moments as well as the one-electron energies on the exchange-correlation energy functionals. We found that, although the orbital and spin magnetic moments increase in magnitude notably when replacing LSDA with GGA, the cancellation results in the total magnetic moments being almost unchanged for all the functionals employed in this work. We also found that the functional dependence of the oneelectron energies is small. Thus, the inclusion of the gradient and relativistic corrections fails to improve the agreement between the calculated and experimental results with respect to the total magnetic moments and the one-electron energies. 
Table I. Lattice constants of $\mathrm{U} X(X=\mathrm{S}, \mathrm{Se}, \mathrm{Te})$ in unit of $\AA$. In the table, the results obtained using the FFLCAO and SFLCAO methods are shown, where those obtained using the latter method are in parentheses. The experimental lattice constants taken from ref. 40 are also shown. The exchange-correlation energy functionals employed in this work are the Perdew-Zunger, PerdewWang, PBEsol, and PBE functionals. Also, for each material, the first and second rows show the results of the calculations without and with the relativistic correction to the LSDA exchange part, respectively.

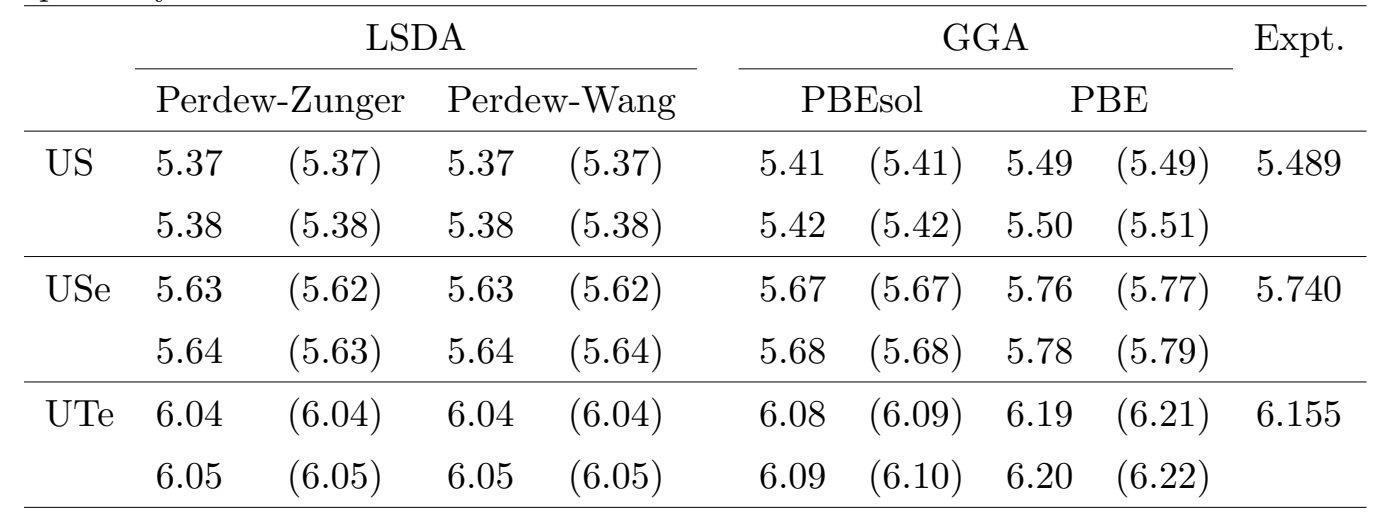

Table II. Bulk moduli of $\mathrm{U} X(X=\mathrm{S}$, Se, Te) in unit of GPa. In the table, the results obtained using the FFLCAO and SFLCAO methods are shown, where those obtained using the latter method are in parentheses. The experimental bulk moduli taken from ref. 43 are also shown. The exchange-correlation energy functionals employed in this work are the Perdew-Zunger, PerdewWang, PBEsol, and PBE functionals. Also, for each material, the first and second rows show the results of the calculations without and with the relativistic correction to the LSDA exchange part, respectively.

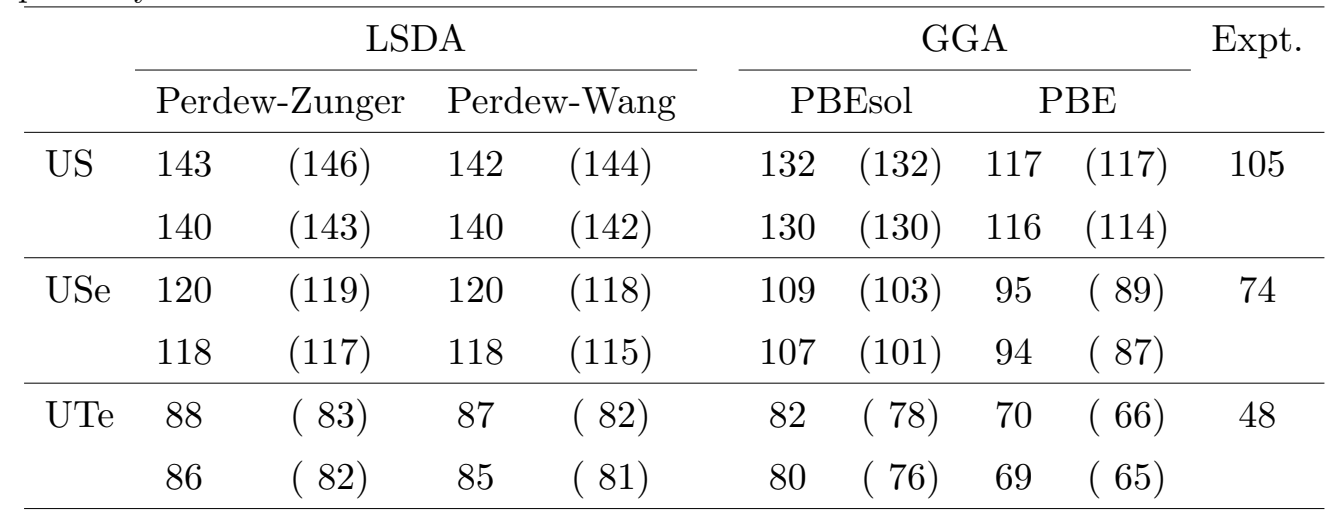


Table III. Cohesive energies of $\mathrm{U} X(X=\mathrm{S}, \mathrm{Se}, \mathrm{Te})$ in unit of eV/atom. In the table, the results obtained using the FFLCAO and SFLCAO methods are shown, where those obtained using the latter method are in parentheses. The experimental cohesive energies taken from ref. 44 are also shown. The exchange-correlation energy functionals employed in this work are the Perdew-Zunger, Perdew-Wang, PBEsol, and PBE functionals. Also, for each material, the first and second rows show the results of the calculations without and with the relativistic correction to the LSDA exchange part, respectively.

\begin{tabular}{|c|c|c|c|c|c|c|c|c|c|}
\hline \multirow{3}{*}{ US } & \multicolumn{4}{|c|}{ LSDA } & \multicolumn{4}{|c|}{ GGA } & \multirow[t]{2}{*}{ Expt. } \\
\hline & \multicolumn{2}{|c|}{ Perdew-Zunger } & \multicolumn{2}{|c|}{ Perdew-Wang } & \multicolumn{2}{|c|}{ PBEsol } & \multicolumn{2}{|c|}{ PBE } & \\
\hline & 7.42 & $(7.44)$ & 7.37 & $(7.38)$ & 7.05 & $(6.96)$ & 6.49 & $(6.43)$ & 5.65 \\
\hline & 7.36 & $(7.37)$ & 7.31 & $(7.32)$ & 6.99 & $(6.90)$ & 6.44 & $(6.42)$ & \\
\hline \multirow[t]{2}{*}{ USe } & 6.70 & $(6.76)$ & 6.65 & $(6.71)$ & 6.38 & $(6.34)$ & 5.84 & $(5.78)$ & 4.99 \\
\hline & 6.65 & $(6.70)$ & 6.60 & $(6.65)$ & 6.33 & $(6.29)$ & 5.80 & $(5.78)$ & \\
\hline \multirow[t]{2}{*}{ UTe } & 5.80 & $(5.95)$ & 5.76 & $(5.90)$ & 5.57 & $(5.64)$ & 5.09 & $(5.13)$ & 4.43 \\
\hline & 5.76 & $(5.90)$ & 5.72 & $(5.86)$ & 5.54 & $(5.59)$ & 5.07 & $(5.14)$ & \\
\hline
\end{tabular}


J. Phys. Soc. Jpn.

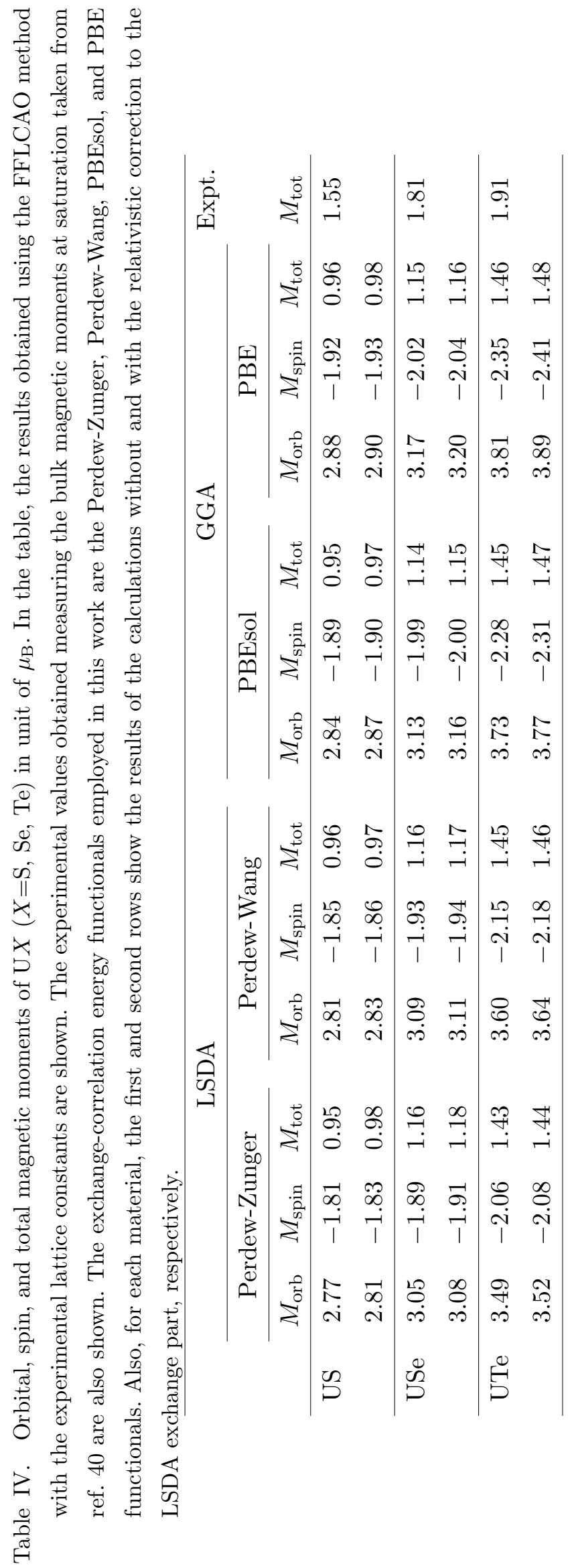




\section{References}

1) Handbook on the Physics and Chemistry of the Actinides, ed. A. J. Freeman and G. H. Lander (North-Holland, Amsterdam, 1985).

2) Electronic Structure: Basic Theory and Practical Methods, R. M. Martin (Cambridge University Press, 2004).

3) P. Söderlind, O. Eriksson, B. Johansson, and J. M. Wills: Phys. Rev. B 50 (1994) 7291.

4) K. N. Kudin, G. E. Scuseria, and R. L. Martin: Phys. Rev. Lett. 89 (2002) 266402.

5) J. S. Olsen, L. Gerward, V. Kanchana, and G. Vaitheeswaran: J. Alloys Comp. 381 (2004) 37.

6) D. Sedmidubský, R. J. M. Konings, and P. Novák: J. Nucl. Mater. 344 (2005) 40.

7) I. D. Prodan, G. E. Scuseria, and R. L. Martin: Phys. Rev. B 73 (2006) 045104.

8) P. F. Weck, E. Kim, N. Balakrishnan, F. Poineau, C. B. Yeamans, and K. R. Czerwinski: Chem. Phys. Lett. 443 (2007) 82.

9) R. Atta-Fynn and A. K. Ray: Phys. Rev. B 76 (2007) 115101.

10) R. A. Evarestov, A. V. Bandura, M. V. Losev, E. A. Kotomin, Y. F. Zhukovskii, and D. Bocharov: J. Comput. Chem. 29 (2008) 2079.

11) F. Jollet, G. Jomard, B. Amadon, J. P. Crocombette, and D. Torumba: Phys. Rev. B 80 (2009) 235109.

12) P. Söderlind and A. Gonis: Phys. Rev. B 82 (2010) 033102.

13) M. S. S. Brooks: J. Magn. Magn. Mater. 29 (1982) 257.

14) M. S. S. Brooks and P. J. Kelly: Phys. Rev. Lett. 51 (1983) 1708.

15) M. S. S. Brooks: J. Phys. F: Phys. 14 (1984) 639.

16) M. S. S. Brooks: J. Phys. F: Phys. 14 (1984) 653.

17) M. S. S. Brooks: J. Phys. F: Phys. 14 (1984) 857.

18) M. S. S. Brooks: Physica 130B (1985) 6.

19) M. S. S. Brooks, B. Johansson, O. Eriksson, and H. L. Skriver: Physica 144B (1986) 1.

20) J. P. Perdew nad Y. Wang: Phys. Rev. B 33 (1986) 8800.

21) J. P. Perdew, J. A. Chevary, S. H. Vosko, K. A. Jackson, M. R. Pederson, D. J. Singh ,and C. Fiolhais: Phys. Rev. B 46 (1992) 6671.

22) J. P. Perdew, K. Burke, and M. Ernzerhof: Phys. Rev. Lett. 77 (1996) 3865.

23) J. P. Perdew, A. Ruzsinszky, G. I. Csonka, O. A. Vydrov, G. E. Scuseria, L. A. Constantin, X. Zhou, and K. Burke: Phys. Rev. Lett. 100 (2008) 136406.

24) P. Haas, F. Tran, and P. Blaha: Phys. Rev. B 79 (2009) 085104.

25) J. Trygg, J. M. Wills, M. S. S. Brooks, B. Johansson, and O. Eriksson: Phys. Rev. B 52 (1995) 2496.

26) T. Kraft, P. M. Oppeneer, V. N. Antonov, and H. Eschrig: Phys. Rev. B 52 (1995) 3561.

27) H. Yamagami: J. Phys. Soc. Jpn. 67 (1998) 3176.

$28)$ E. M. Collins, N. Kioussis, S. P. Lim, and B. R. Cooper: J. Appl. Phys. 85 (1999) 6226.

29) T. Shishidou and T. Oguchi: Phys. Rev. B 62 (2000) 11747.

30) V. N. Antonov, B. N. Harmon, O. V. Andryushchenko, L. V. Bekenev, and A. N. Yaresko: Low Temp. Phys. 30 (2004) 305.

31) S. Suzuki, T. Ariizumi, and M.-F. Li: J. Phys. Soc. Jpn. 78 (2009) 074715.

32) S. Suzuki and H. Ohta: J. Phys. Soc. Jpn. 79 (2010) 074703. 
33) A. O. Shorikov, J. E. Medvedeva, A. I. Poteryaev, V. V. Mazurenko, and V. I. Anisimov: JETP Lett. 91 (2010) 486.

34) S. Suzuki and K. Nakao: J. Phys. Soc. Jpn. 68 (1999) 1982.

35) S. Suzuki and K. Nakao: J. Phys. Soc. Jpn. 69 (2000) 532.

36) J. P. Perdew and A. Zunger: Phys. Rev. B 23 (1981) 5048.

37) J. P. Perdew and Y. Wang: Phys. Rev. B 45 (1992) 13244.

38) D. M. Ceperley and B. J. Alder: Phys. Rev. Lett. 45 (1980) 566.

39) A. H. MacDonald: J. Phys. C: Solid State Phys. 16 (1983) 3869.

40) J. M. Fournier and R. Troć: in Handbook on the Physics and Chemistry of the Actinides, ed. A. J. Freeman and G. H. Lander (North-Holland, Amsterdam, 1985) Vol. 2, p. 29.

41) L.-K. Hua and Y. Wang: Applications of Number Theory to Numerical Analysis (Springer-Verlag, Berlin, 1981).

42) F. D. Murnaghan: Proc. Natl. Acad. Sci. USA 30 (1944) 244.

43) U. Benedict: J. Alloys Comp. 223 (1995) 216.

44) Y. Baskin and S. D. Smith: J. Nucl. Mater. 37 (1970) 209.

45) U. von Barth and L. Hedin: J. Phys. C 5 (1972) 1629. 